\title{
ELABORAÇÃO DE QUEIJO DE COALHO A PARTIR DE LEITE PASTEURIZADO E INOCULADO COM Streptococcus thermophyllus E Lactobacillus bulgaricus: ASPECTOS BIOQUÍMICO E SENSORIAL
}

\author{
MÁRCIA HELENA PORTELA LIMA * \\ FRANCISCO JOSÉ SIQUEIRA TELLES ** \\ BENEMÁRIA ARAÚJO MACEDO *** \\ SELENE DAIHA BENEVIDES ****
}

\begin{abstract}
Queijos de coalho elaborados a partir de leite tratado termicamente e inoculado a $1 \%$ com cultura termofílica (Streptococcus thermophyllus e Lactobacillus bulgaricus) foram comparados com queijos obtidos tradicionalmente. Considerou-se no estudo a intensidade de cura e a aceitabilidade do produto. A maturação foi realizada a temperatura ambiente $\left(29^{\circ} \mathrm{C}\right)$ e em câmara fria $\left(12^{\circ} \mathrm{C}\right)$ durante dez dias. Os queijos apresentaram índices de maturação próximos e valores ligeiramente maiores para aqueles inoculados com fermento lático e maturação a temperatura ambiente. A média de aceitabilidade (ao nível de $5 \%$ de significância) evidenciou a possibilidade de obtenção de queijo de coalho a partir de leite pasteurizado e inoculado com S. thermophyllus e $L$. bulgaricus. As médias atribuídas pelos provadores indicaram certa preferência por este queijo, quando maturado a temperatura ambiente.
\end{abstract}

\section{INTRODUÇÃO}

O queijo de coalho é o mais tradicional dos queijos produzidos no nordeste brasileiro, sendo amplamente fabricado e consumido em todo o Estado do Ceará. Seu nome deve-se ao fato de ter sido tradicionalmente elaborado com leite coagulado pela ação do coalho animal: pedaços do estômago de pequenos animais (mocó, preá, cabrito, bezerro), que devidamente preparados são conhecidos por abomasun ou coagulador (AQUINO, 1983).

Apesar de sua importância econômica e grande popularidade na região, a fabricação de queijo de coalho não conta com tecnologia apropriada para a melhoria de sua qualidade. É necessário que o processo tradicional, geralmente artesanal e a partir de leite cru, acompanhe a evolução das técnicas queijeiras mediante modificações já utilizadas na tecnologia de outros queijos nacionais.

* Pesquisadora do Núcleo de Tecnologia Industrial (NUTEC), Fortaleza, Ceará

** Professor Adjunto IV, Universidade Federal do Ceará (UFC).

*** Pesquisadora, Universidade Estadual do Ceará (UECE).

$\star * * *$ Engenheira de Alimentos, UFC. 
O uso de leite pasteurizado e o emprego de fermento lático têm contribuído de forma significativa para a melhoria da qualidade dos queijos em geral no Brasil (OLIVEIRA, 1987). A pasteurização do leite para o fabrico de queijo dispensa qualquer comentário, quando se reconhece sua importância com relação à Saúde Pública, além de vantagens tecnológicas como diminuição das fermentações anormais, maior rendimento e melhor textura (FURTADO, 1989). O fermento lático selecionado, por sua vez, promove resultado tecnológico excelente ao produzir fermentação mais controlada e, nos queijos é responsável pela acidificação e produção de flavor (NIELSEN \& ULLUM, 1989, VIEIRA, 1981).

Talvez a mais importante consideração na manufatura de queijos seja a obtenção de produto aceitável sob o ponto de vista de flavor e textura (DAVIES \& LAW, 1984). A proteólise nos queijos por ação microbiana e do próprio coalho exerce considerável influência na textura e sabor do produto sendo medida pelo índice de maturação, o qual constitui-se em importante parâmetro de qualidade dos queijos (JOHNSON, 1988).

O fermento lático, composto pela cultura do iogurte (Streptococcus thermophillus e Lactobacillus bulgaricus), é facilmente encontrado no comércio. Por ser termofílico é utilizado com excelente resultado na manufatura de queijo, cuja massa sofre considerável aquecimento durante a fabricação (EMALDI, 1989).

O presente trabalho visou a comparação de queijos de coalho, elaborados a partir de leite tratado termicamente e inoculado com cultura termofílica, com queijos obtidos tradicionalmente, em relação a intensidade de cura e aceitabilidade do produto. Empregou-se a técnica da pasteurização lenta $\left(65{ }^{\circ} \mathrm{C} 30^{\prime}\right)$ por tratar-se de método de fácil adoção em termos de disponibilidade e custo de equipamentos. Procurou-se averiguar a compatibilidade das modificações introduzidas nas condições de fabricação do queijo na hipótese de que as mesmas permitissem a obtenção de produto com melhor qualidade, mas dentro de suas características tradicionais.

\section{MATERIAL E MÉTODOS}

Os queijos foram elaborados por processos baseados na técnica de produção mais comum do Estado, diferenciados no entanto, pelo tratamento inicial dado ao leite (com e sem pasteurização/inoculação) e pelas condições de maturação (em câmara pré-condicionada a $12{ }^{\circ} \mathrm{C}$ e a temperatura ambiente). Os 4 (quatro) tratamentos foram realizados com 5 (cinco) repetições cada. 


\subsection{MATERIAL}

\subsubsection{Leite}

Como matéria-prima utilizou-se leite cru integral, obtido de vacas mestiças (zebu-holandês), apresentando valores de 17 a 20 para acidez, expressos em graus DORNIC e de 3,6 a 3,8\% para gordura.

\subsubsection{Agente Coagulante}

Usou-se suspensão feita a partir da dissolução de $3,1 \mathrm{~g}$ de coalho em pó, comercial (marca "HA-LA") em $150 \mathrm{~mL}$ de água, juntamente com $20 \mathrm{~g}$ de sal refinado comercial, para $50 \mathrm{~L}$ de leite a ser coagulado.

\subsubsection{Cultura Lática}

Empregou-se a cultura da Christian Hansen Laboratory, composta de Lactobacillus bulgaricus e Streptococcus thermophillus na forma líquida, obtida diretamente da cultura intermediária industrial para fabricação de iogurte da Companhia de Laticínios do Ceará (CILA).

\subsubsection{Cloreto de Cálcio}

Utilizou-se solução hidrossolúvel a 40\%, marca PASTORINHA.

\subsubsection{Cloreto de Sódio}

Empregou-se sal comercial, refinado e iodado, marca MARLIN.

\subsection{MÉTODOS DE PRODUÇÃO DOS QUEIJOS}

As etapas básicas utilizadas na fabricação dos queijos, juntamente com as variações que originaram os 4 (quatro) tratamentos, estão representadas no fluxograma da Figura 1.

\subsection{MÉTODOS DE ANÁLISE}

Os queijos foram analisados após 10 dias de fabricação, considerando-se este tempo como o período médio de consumo do produto pela população. 


\section{FIGURA 1 - FLUXOGRAMA DAS ETAPAS DE FABRICAÇÃO DOS QUEIJOS DE COALHO}

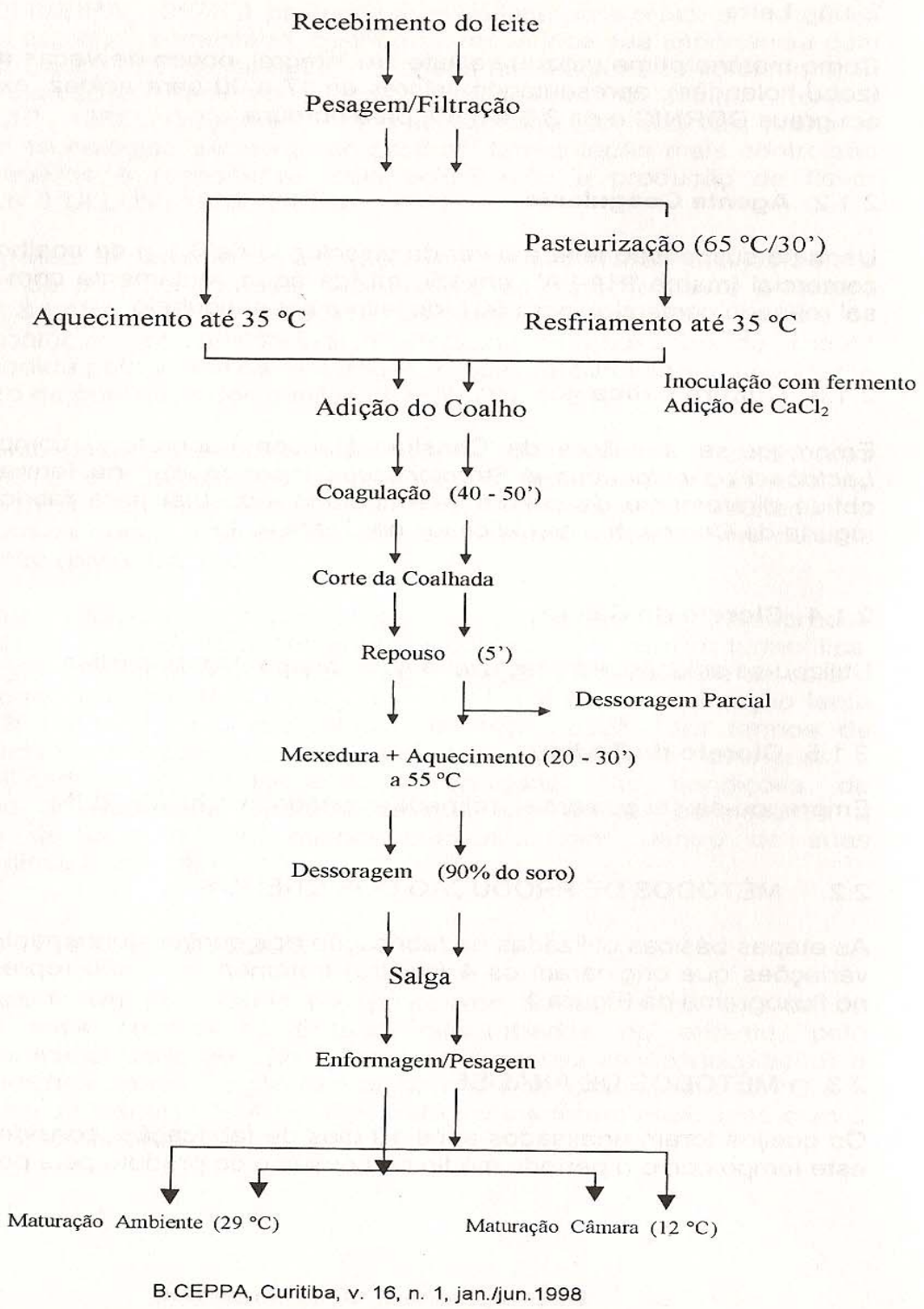




\subsection{1 Índice de maturação (IM)}

Obteve-se o índice de maturação pela relação percentual entre os teores de proteína solúvel (extração pelo método KOSIKOWSKI e determinação do nitrogênio pelo método de KJELDAHL) e proteína total (LANARA, 1981).

\subsubsection{Análise sensorial}

A avaliação sensorial dos queijos foi feita a partir do teste de aceitação, com 31 provadores não treinados, utilizando-se escala hedônica de 9 pontos (nota 9 significando "gostei extremamente" e 1 "desgostei muitíssimo").

As amostras em forma de cubos de 1 a $2 \mathrm{~cm}^{3}$ foram servidas a temperatura ambiente e codificadas com números aleatórios de três dígitos (SMITH, 1985).

\section{RESULTADOS E DISCUSSÃO}

Os resultados dos índices de maturação dos queijos, com as médias obtidas pela relação percentual entre as proteínas solúveis e totais, encontram-se na Tabela 1.

A análise estatística revelou efeito do tipo do queijo e da temperatura para o índice de maturação $(P<0,000)$. Os valores foram ligeiramente maiores para os queijos inoculados com o fermento e maturação feita a temperatura ambiente, mas a diferença só foi significativa com relação ao local de maturação para o queijo feito com leite cru. Em ambos os tipos de queijo, os valores encontrados foram considerados baixos revelando que a degradação das proteínas (caseínas) foi limitada durante o período de 10 dias. O teor de sal relativamente alto em queijos pode ser condicionante da proteólise (VEISSEYRE, 1988).

Os resultados da análise sensorial dos queijos encontram-se na Tabela 2, com as notas conferidas pelos provadores. Em função das médias alcançadas considerou-se aceitáveis os queijos na faixa de aprovação situada entre "gostei levemente" e "gostei". Ao nível de 5\% de significância, o teste de Friedman indicou que não houve diferença entre os queijos nos quatro tratamentos considerados. Verificou-se, no entanto, que a média obtida pelo queijo feito com leite pasteurizado/inoculado, maturado a temperatura ambiente, apresentou coeficiente de variação (CV) inferior às demais médias, evidenciando certa preferência por este queijo. A combinação da maciez observada nos queijos pasteurizados, com o sabor 
mais intenso constatado nos queijos maturados a temperatura ambiente, pode explicar maior aceitação deste queijo em relação aos demais (DAVIES \& LAW, 1984).

\section{TABELA 1 - ÍNDICE DE MATURAÇÃO (PROTEÍNA SOLÚVELI PROTEÍNA TOTAL X 100) DOS QUEIJOS DE COALHO AOS 10 DIAS DE MATURAÇÃ̃O EM DIFERENTES TEMPERATURAS}

\begin{tabular}{lrrrr}
\hline Local de Maturação & \multicolumn{2}{c}{ Ambiente $\left(29^{\circ} \mathrm{C}\right)$} & \multicolumn{2}{c}{ Câmara $\left(12^{\circ} \mathrm{C}\right)$} \\
\hline $\begin{array}{l}\text { Tipo de Coalho } \\
\text { Análise }\end{array}$ & $*$ Qc & ${ }^{* *}$ Qp & Qc & Qp \\
\hline & & & & \\
Proteína Total (\%) & 25,57 & 25,22 & 24,23 & 24,62 \\
Proteína Solúvel (\%) & 2,64 & 2,67 & 2,24 & 2,42 \\
Índice de Maturação & 10,32 & 10,59 & 9,24 & 9,83 \\
& & & & \\
\hline
\end{tabular}

* Queijo feito a partir de leite cru.

** Queijo feito a partir de leite pasteurizado/inoculado com S. thermophillus e L. bulgaricus.

TABELA 2 - MÉDIA DAS NOTAS DO TESTE DE ACEITAÇÃO DOS QUEIJOS DE COALHO AOS 10 DIAS DE MATURAÇÃO EM DIFERENTES TEMPERATURAS

\begin{tabular}{lcccc}
\hline TIPO DE QUEIJO & 1 & 2 & 3 & 4 \\
\hline Média & 6,71 & 6,68 & 7,68 & 6,94 \\
\hline $\begin{array}{l}\text { Coeficiente de } \\
\text { variação }\end{array}$ & $24,90 \%$ & $24,80 \%$ & $8,99 \%$ & $26,63 \%$ \\
\hline
\end{tabular}

$1=$ Queijo feito com leite cru maturado a temperatura ambiente $\left(29^{\circ} \mathrm{C}\right)$

$2=$ Queijo feito com leite cru maturado em câmara fria $\left(12^{\circ} \mathrm{C}\right)$

3 = Queijo feito com leite pasteurizado/inoculado com S. thermophillus L. bulgaricus, maturado a temperatura ambiente $\left(29^{\circ} \mathrm{C}\right)$.

4 = Queijo feito com leite pasteurizado/inoculado com S. thermophillus e L. bulgaricus, maturado em câmara fria $\left(12^{\circ} \mathrm{C}\right)$.

\section{CONCLUSÃO}

Os valores encontrados para os índices de maturação dos queijos sugerem que o fermento lático pode ter sido utilizado em baixa quantidade ou 
apresentar pouca atividade proteolítica. Ao mesmo tempo denotam a importância da microbiota do leite cru em termos de cura, ao apresentar valores próximos aos da cultura lática selecionada. É possível a elaboração de queijo de coalho com o emprego da cultura de $\mathrm{S}$. thermopillus e L. bulgaricus a partir de leite pasteurizado $\left(65^{\circ} \mathrm{C} 30^{\prime}\right)$, visto que os mesmos não apresentaram diferença, em termos de níveis de aceitação, dos queijos feitos tradicionalmente com leite cru. As notas atribuídas aos produtos no teste sensorial indicaram melhor aceitação dos queijos elaborados com leite pasteurizado/inoculado (S.thermophillus e $L$. bulgaricus), maturados a temperatura ambiente.

\begin{abstract}
Coagulum type cheeses made from milk heat-treated (LTLT pasteurization) and inoculated with $1 \%$ starter (Streptococcus thermophillus and Lactobacillus bulgaricus) were compared to those cheeses traditionally obtained from raw milk concerning the intensity of cure and acceptability. The cheese ripening was realized at room temperature $\left(29^{\circ} \mathrm{C}\right)$ and in cold chamber $\left(12^{\circ} \mathrm{C}\right)$ during ten days. The cheeses presented approximate ripening rates, however values were slightly higher for those inoculated with lactic ferment and with the ripening done at room temperature. The cheeses were considered equal in a $5 \%$ level of significance. It means that it is possible to obtain coagulum cheeses from pasteurized milk with the thermophylic culture used. The sensory tests indicated a preference for those cheeses made from pasteurized/inoculated milk ripened at room temperature.
\end{abstract}

\title{
REFERÊNCIAS BIBLIOGRÁFICAS
}

1 AQUINO, F. T. M. Produção do queijo de coalho no Estado da Paraíba: acompanhamento das carterísticas físico-químicas do processamento. João Pessoa, 1983. 74 p. Dissertação (Mestrado em Ciência e Tecnologia de Alimentos) - Universidade Federal da Paraíba.

2 BRASIL. Ministério da Agricultura. Laboratório Nacional de Referência Animal. Métodos analíticos oficiais para controle de produtos de origem animal e seus ingredientes: métodos físicos e químicos. Brasília, 1981. v. 2.

3 DAVIES, F. L., LAW, B. A. Advances in the microbiology and biochemistry of cheese and fermented milk. London : Elsevier Applied Science, 1984. $260 \mathrm{p}$.

4 EMALDI, G. C. Queijos italianos. In: SEMINÁRIO BRASILEIRO DE QUEIJOS ITALIANOS, 11., out. 1983. Sinopse... Juiz de Fora : EPAMIG, 1989. $45 \mathrm{p}$.

5 FURTADO, M. M. Fundamentos básicos da fabricação de queijos semi-cozidos. Viçosa : UFV, 1989. $164 \mathrm{p}$. 
6 JOHNSON, M. E. Cheese chemistry. In: WONG, N.P., JENNESS, R., KEENEY, M. et al. Fundamentals of dairy chemistry. 3. ed. New York : Van Nostrand Reinhold, 1988. p. 634-654.

7 KOSIKOWSKI, F.C. Cheese and fermented milk foods. New York : [s.n.], 1982. p. 572.

8 NIELSEN, E. W., ULLUM, J. A. Dairy technology 2. Denmark : Danish Turnkey Dairies, 1989. 286 p.

9 OLIVEIRA, J.S. Como fazer queijos: fundamentos tecnológicos. Rio de Janeiro : Tecnoprint, 1987. $146 \mathrm{p}$.

10 SMITH, L. F. Avaliações sensoriais de queijo. Rev. Inst. Latic. Cândido Tostes, Juiz de Fora, v. 40, n. 239, p. 106-118, 1985.

11 VEISSEYRE, Roger. Lactologia técnica. Zaragoza : Acribia, 1988. $629 \mathrm{p}$.

12 VIEIRA, S.D.A. A utilização de culturas láticas na indústria de laticínios. Rev. Inst. Latic. Cândido Tostes, Juiz de Fora, v. 36, n. 215, p. 29-38, 1981. 